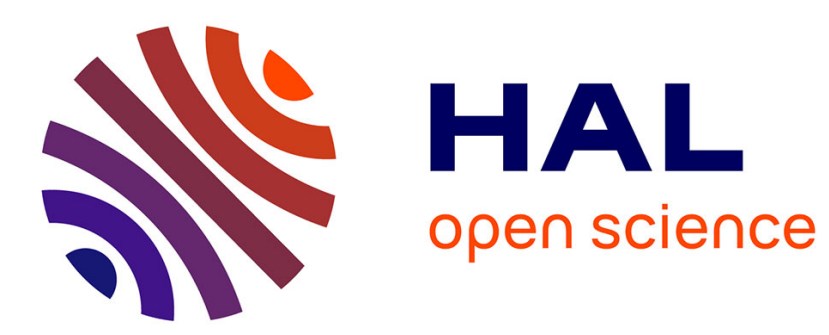

\title{
On weakly uniform integer additive set-indexers of graphs
}

K. A Germina, Sudev Naduvath

\section{To cite this version:}

K. A Germina, Sudev Naduvath. On weakly uniform integer additive set-indexers of graphs. International Mathematical Forum, 2013, 8, pp.1827-1834. 10.12988/imf.2013.310188 . hal-02099206

\section{HAL Id: hal-02099206 \\ https://hal.science/hal-02099206}

Submitted on 7 May 2019

HAL is a multi-disciplinary open access archive for the deposit and dissemination of scientific research documents, whether they are published or not. The documents may come from teaching and research institutions in France or abroad, or from public or private research centers.
L'archive ouverte pluridisciplinaire HAL, est destinée au dépôt et à la diffusion de documents scientifiques de niveau recherche, publiés ou non, émanant des établissements d'enseignement et de recherche français ou étrangers, des laboratoires publics ou privés. 
International Mathematical Forum, Vol. 8, 2013, no. 37, 1827 - 1834

HIKARI Ltd, www.m-hikari.com

http://dx.doi.org/10.12988/imf.2013.310188

\title{
On Weakly Uniform Integer Additive Set-Indexers of Graphs
}

\author{
K. A. Germina \\ Department of Mathematics \\ School of Mathematical \& Physical Sciences \\ Central University of Kerala \\ Kasaragod, Kerala, India \\ N. K. Sudev \\ Department of Mathematics \\ Vidya Academy of Science \& Technology \\ Thrissur, Kerala, India
}

Copyright (C) 2013 K. A. Germina and N. K. Sudev. This is an open access article distributed under the Creative Commons Attribution License, which permits unrestricted use, distribution, and reproduction in any medium, provided the original work is properly cited.

\begin{abstract}
We have the notion of set-indexers, integer additive set-indexers and $k$-uniform integer additive set-indexers of graphs. In this paper, we initiate a study of the graphs which admit $k$-uniform integer additive set-indexers and introduce the notion of weakly uniform integer additive set-indexers and arbitrarily uniform integer additive set-indexers and provide some useful results on these types of set-indexers.
\end{abstract}

\section{Mathematics Subject Classification: 05C78}

Keywords: $k$-uniform Integer Additive Set-Indexers, Weakly $k$-uniform Integer Additive Set-Indexers, Arbitrarily $k$-uniform Integer Additive Set-Indexers 


\section{Introduction}

For all terms and definitions, not defined specifically in this paper, we refer to [7]. Unless mentioned otherwise, all graphs considered here are simple, finite and have no isolated vertices.

The set-labeling of a graph is defined in [1] as follows. Let $G$ be a $(p, q)$ graph and let $X, Y, Z$ be non-empty sets. Then the functions $f: V(G) \rightarrow 2^{X}$, $f: E(G) \rightarrow 2^{Y}$ and $f: V(G) \cup E(G) \rightarrow 2^{Z}$ are called the set-assignments of vertices, edges and elements of $G$ respectively. By a set-assignment of a graph, we mean any one of them. A set-assignment is called a set-labeling if it is injective.

Definition 1.1 [1] For a $(p, q)$ - graph $G=(V, E)$ and a non-empty set $X$ of cardinality $n$, a set-indexer of $G$ is defined as an injective set-valued function $f: V(G) \rightarrow 2^{X}$ such that the function $f^{\oplus}: E(G) \rightarrow 2^{X}-\{\emptyset\}$ defined by $f^{\oplus}(u v)=f(u) \oplus f(v)$ for every $u v \in E(G)$ is also injective, where $2^{X}$ is the set of all subsets of $X$ and $\oplus$ is the symmetric difference of sets.

Theorem 1.2 [1] Every graph has a set-indexer.

Definition 1.3 Let $N_{0}$ denote the set of all non-negative integers. For all $A, B \subseteq N_{0}$, the sum of these sets is denoted by $A+B$ and is defined by $A+B=\{a+b: a \in A, b \in B\}$.

Definition 1.4 [6] An integer additive set-indexer (IASI, in short) is defined as an injective function $f: V(G) \rightarrow 2^{N_{0}}$ such that the induced function $g_{f}: E(G) \rightarrow 2^{N_{0}}$ defined by $g_{f}(u v)=f(u)+f(v)$ is also injective.

Definition 1.5 The cardinality of the labeling set of an element (vertex or edge) of a graph $G$ is called the set-indexing number of that element.

Definition 1.6 [6] An IASI is said to be $k$-uniform if $\left|g_{f}(e)\right|=k$ for all $e \in E(G)$. That is, a connected graph $G$ is said to have a k-uniform IASI if all of its edges have the same set-indexing number $k$. In particular, we say that a graph $G$ has an arbitrarily $k$-uniform IASI if $G$ has a $k$-uniform IASI for every positive integer $k$.

In [6], a study of a special type of 1-uniform IASI sum square graphs and in[2], a characterisation of 2-uniform IASI were established. The most important result given in [2] is the following.

Theorem 1.7 [2] A graph G has a 2-uniform IASI if and only if it is a bipartite graph. 
The motivation for the study presented in this paper is from the results in [6] and [2]. As a generalisation of 1-uniform and 2-uniform IASIs, in section 2 , we introduce the notion of weakly $k$-uniform IASI and arbitrarily $k$-uniform IASI and prove the conditions for a connected graph to admit a weakly or arbitrarily $k$-uniform IASI, where $k$ is a positive integer. Finally, in section 3, some illustrations are also provided.

\section{Uniform Integer Additive Set-Indexers}

During this study our first attempt is to analyse the set-indexing number of an edge of a graph $G$ and to study its relation with the set-indexing number of the end vertices of that edge. As a result, we establish the following results regarding the cardinality of $A+B$ defined in Definition 1.3. If either $A$ or $B$ is countably infinite, then clearly $A+B$ is also countably infinite and hence the study of the cardinality of $A+B$ trivial. Hence we restrict our discussion on finite sets $A$ and $B$. We denote the cardinality of a set $A$ by $|A|$.

Lemma 2.1 Let $A, B \subseteq N_{0}$. Then $\max (|A|,|B|) \leq|A+B| \leq|A||B|$.

Proof: Let $A, B \subseteq N_{0}$. Also let $|A|=m,|B|=n$. Assume either $A$ or $B$ is a singleton set. Without loss of generality, let $A$ be a singleton set, say $A=\{q\}$ where $q$ is a non-negative integer. That is, $m=1$. Therefore, $A+B=$ $\left\{b_{1}+q, b_{2}+q, . . \cdots b_{n}+q\right\}$. Hence $|A+B|=n$. Similarly, if $|B|=1$, then $|A+B|=m$. Hence, $\max (|A|,|B|) \leq|A+B|$. If neither $A$ nor $B$ is singleton, then define $h: A \times B \rightarrow A+B$ defined by, $h(a, b)=a+b ; a \in A, b \in B$. Clearly, $h$ is injective and hence $|A+B| \leq|A||B|$. The equality holds when the function $h: A \times B \rightarrow A+B$ is a bijection. From the above two cases, we have $\max (|A|,|B|) \leq|A+B| \leq|A||B|$.

Remark 2.2 Due to Lemma 2.1, given an integer additive set-indexer $f$ of a graph $G, \max (|f(u)|,|f(v)|) \leq\left|g_{f}(u v)\right|=|f(u)+f(v)| \leq|f(u)||f(v)|$, where $u, v \in V(G)$.

The characteristics of the IASIs with the property $|A+B|=\max (|f(u)|$, $|f(v)|)$ is of special interest. Hence we have the following definition.

Definition 2.3 An IASI $f$ of a graph $G$ is called a weak IASI if $\left|g_{f}(u v)\right|=$ $\max (|f(u)|,|f(v)|)$ for all $u, v \in V(G)$.

It is observed that if $\left|g_{f}(u v)\right|=\max (|f(u)|,|f(v)|) \forall u, v \in V(G)$ holds for every edge $u v$ of $G$, it is necessary that one of its end vertices is labeled by a singleton set. Hence we define a weakly $k$-uniform IASI as follows. 
Definition 2.4 A $k$-uniform IASI which assigns only singleton sets and $k$ element sets to the vertices of a given graph $G$ is called a weakly $k$-uniform IASI.

Theorem 2.5 Every tree admits a weakly k-uniform IASI.

Proof: All the sets under consideration are subsets of $N_{0}$. Let $T$ be the given tree and $k$ be an arbitrary positive integer. If $k=1$, assign distinct singleton sets to every vertex of $T$. Then clearly, every edge of $T$ also has singleton sets as labeling sets. Therefore, $T$ is 1-uniform.

Now let $k>1$.

Step-1: Let $u_{1}, u_{2}, \cdots u_{r}$ be the pendant vertices of $T$. Assign the singleton sets $\{i\}$ to the pendant vertices $u_{i}, 1 \leq i \leq r$. Let $\left\{v_{1}, v_{2}, \cdots v_{s}\right\}$ be the set of internal vertices of $T$ which are adjacent to some of $u_{i}, 1 \leq i \leq s$. Assign the $k$-element set $\{j, j+1, j+2, \cdots j+(k-1)\}$ to each vertex $v_{j}, 1 \leq j \leq s$. Then, by Theorem 2.1, each edge $u_{i} v_{j}$ has the set-indexing number $k$. Note that no $u_{i}$ can be adjacent to two internal vertices which are adjacent to each other, since otherwise, we have a contradiction to the acyclic property of $T$.

Step-2: Let $\left\{w_{1}, w_{2}, \cdots w_{l}\right\}$ be the set of internal vertices which are adjacent to some of $v_{j}, 1 \leq j \leq l$, where no $w_{n}$ can be adjacent to any pendant vertices. Assign singleton sets $\{r+n\}$ to each $w_{n}, 1 \leq n \leq l$ so that each edge $v_{j} w_{n}$ has the set-indexing number $k$.

The succeeding steps, the vertices that are adjacent to the just preceding labeled vertices alternately by distinct $k$-element sets other than the already labelled sets and distinct singleton sets, for a finite number of times, we get a weakly $k$-uniform IASI to the tree $T$.

Remark 2.6 A path $P_{n}$ can be considered as a tree which has two pendant vertices. Hence by Theorem 2.5, $P_{n}$ admits an arbitrary $k$-uniform IASI.

Theorem 2.7 Every even cycle $C_{2 n}$ has a weakly $k$-uniform IASI.

Proof: Let $C_{2 n}=v_{1} v_{2} v_{3} \cdots v_{2 n} v_{1}$ be a cycle of even length. Assign distinct singleton subsets of $N_{0}$ to the vertices $v_{1}, v_{3}, \ldots v_{2 n-1}$ and assign distinct $k$ element subsets of $N_{0}$ to the vertices $v_{2}, v_{4}, \cdots v_{2 n}$ of $C_{2 n}$. Then by Theorem 2.1 , each edge $v_{i} v_{j}$ has the set-indexing number $k$. Hence an even cycle has an arbitrary $k$-uniform IASI.

Theorem 2.8 No odd cycle $C_{n}$ has a weakly $k$-uniform IASI.

Proof: By the definition of weakly $k$-uniform IASI, for each edge $v_{i} v_{i+1}$ in $C_{n}$, one of $v_{i}$ (or $v_{i+1}$ ) should necessarily be labeled by a singleton set and $v_{i+1}$ (or $v_{i}$ ) with a $k$-element set. Since $n$ is odd, $n=2 m+1$ for some non-negative 
integer $m$. Let $C_{n}=v_{1} v_{2} v_{3} \cdots v_{2 m+1} v_{1}$ be a cycle of odd length. Assign distinct singleton subsets of $N_{0}$ to the vertices $v_{1}, v_{3}, \ldots v_{2 m-1}$ and assign distinct $k$ element subsets of $N_{0}$ to the vertices $v_{2}, v_{4}, \cdots v_{2 m}$ of $C_{n}$. Then by Theorem 2.1 , each edge $v_{i} v_{j}$ where $i, j \leq 2 n$, has the set-indexing number $k$. Now if we assign a singleton set to the vertex $v_{2 m+1}$, then the edge $v_{2 m+1} v_{0}$ has the set-indexing number 1 . If we assign a $k$-element set to $v_{2 m+1}$, then the edge $v_{2 m} v_{2 m+1}$ has the set-indexing number greater than $k$. Hence no odd cycle admits a weakly $k$-uniform IASI.

Invoking the above results, the existence of a weakly $k$-uniform IASI implies the bipartiteness of the given graph.

Theorem 2.9 Let $k$ be any positive integer. A bipartite graph $G$ admits a weakly $k$-uniform IASI.

Proof: Let $G=\left(X_{1}, X_{2}\right)$ be bipartite graph. Then assign distinct singleton sets to the vertices in $X_{1}$ and distinct $k$-element sets to $X_{2}$ so that every edge of $G$ has the set-indexing number $k$. Therefore, $G$ admits a weakly $k$-uniform IASI.

Theorem 2.10 For any positive integer $k>1$, a graph $G$ admits a weakly $k$-uniform IASI if and only if $G$ is bipartite.

Proof: Proof of the necessary part follows from 2.9. Conversely, assume that $G$ admits a weakly $k$-uniform IASI. That is, every edge of $G$ has the setindexing number $k$. Since $G$ admits a weakly $k$-uniform IASI, one end vertex of all edges of $G$ should necessarily be labeled by distinct singleton sets and the other end vertex by distinct $k$-element sets. Let $X_{1}=\left\{u_{1}, u_{2}, u_{3}, \cdots, u_{r}\right\}$ be the set of vertices which have been labeled by distinct singleton sets and let $X_{2}=\left\{v_{1}, v_{2}, v_{3}, \cdots v_{s}\right\}$ be the set of vertices which have been labeled by $k$-element sets. Since $k>1$, both $X_{1}$ and $X_{2}$ are non-empty and no two vertices of $X_{1}$ (and $X_{2}$ ) can be adjacent to each other. That is, $\left(X_{1}, X_{2}\right)$ is a bipartition of the vertex set of $G$.

Next we establish the existence of $k$-uniform IASIs to bipartite graphs in the following theorem.

Theorem 2.11 A bipartite graph $G$ admits a $k$-uniform IASI.

Proof: Let $G$ be a bipartite graph. Let $\left(X_{1}, X_{2}\right)$ be the bipartition of $V(G)$. Let $X=\left\{v_{1}, v_{2}, v_{3}, \cdots, v_{l}\right\}$ and $Y=\left\{v_{l+1}, v_{l+2}, v_{l+3}, \cdots, v_{r}\right\}$. Let $m, n, d$ be positive integers with $m, n>1$ and $d \geq 1$. Now assign the set $\{i, i+d, i+2 d, \cdots, i+(m-1) d\}$ to the vertex $v_{i}, 1 \leq i \leq l$ in $X_{1}$. Let $v_{l+j}, j \geq 1$, be a vertex in $X_{2}$ which is adjacent to a vertex $v_{i}$. Now assign the set $\{l+j, l+j+d, l+j+2 d, \cdots, l+j+(n-1) d\}$ to the vertex $v_{l+j}$. Now the edge 
$v_{i} v_{l+j}$ has the set-indexer $\{i+j+l, i+j+l+d, i+j+l+2 d, \cdots i+j+l+(n-$ 1) $d, i+j+l+d, i+d+j+l+d, i+d+j+l+2 d, \cdots i+d+j+l+(n-1) d, \cdots, i+$ $(m-1) d+j+l, i+(m-1) d+j+l+d, \cdots, i+(m-1) d+j+d+(n-1) d\}$. That is, the set-indexer of $v_{i} v_{l+j}$ is $\{i+j+l, i+j+l+d, i+j+l+2 d, \cdots, i+j+l+(m-$ 1) $d, i+j+l+m d, i+j+l+(m+1) d, i+j+l+(m+2) d, \cdots, i+j+l+(m+n-2) d\}$. Therefore, the set-indexing number of $u_{i} v_{l+j}$ is $m+n-1$. Since $v_{i}$ and $v_{l+j}$ are arbitrary elements of $X_{1}$ and $X_{2}$ respectively, the above argument can be extended to all edges in $G$. That is, all edges of $G$ can be assigned by a $k$-element set, where $k=m+n-1$. Therefore, $G$ admits a $k$-uniform IASI.

Theorem 2.12 A complete graph $K_{n}, n>2$ does not admit a weakly $k$ uniform IASI for any positive integer $k>1$.

Proof: Let $k>1$ be a positive integer. If possible, let $K_{n}$ admits a weakly $k$-uniform IASI. Then its vertices are assigned by either singleton sets or $k$ element sets. As explained in the proof of Theorem 2.10, we get a bipartition $\left(X_{1}, X_{2}\right)$ of the vertex set of $K_{n}$. This is a contradiction to the fact that no complete graph other than $K_{2}$ is bipartite. Therefore, $K_{n}, n>2$ does not admit a weakly $k$-uniform IASI.

It is interesting to check whether implications of the existence of a weakly $k$ uniform IASI to the existence of an arbitrary $k$ - uniform IASI. For any positive integer $k$, if there exists a weakly $k$-uniform IASI for a graph $G$, then each of its vertices is labeled by either a singleton set or by a $k$-element set and all edges of $G$ have the set-indexing number $k$. Therefore, $G$ has a $k$-uniform IASI. Since $k$ is an arbitrary positive integer, we arrive at the following theorem.

Theorem 2.13 A graph which has a weakly $k$-uniform IASI admits an arbitrarily k-uniform IASI.

Invoking Theorem 2.13, every path, even cycle and tree have an arbitrarily $k$-uniform IASI. Hence we can write the following theorem.

Theorem 2.14 Every bipartite graph admits an arbitrarily $k$-uniform IASI, where $k$ is a positive integer.

We have already established the existence of $k$-uniform IASIs to bipartite graphs for any positive integer $k$. Hence we shall look in to the existence of such uniform IASIs for other connected graphs.

It can be observed that the completely bipartite graph $K_{m, n}$ admits a $k$ uniform IASI for any positive integer $k$. For any given positive integer $k \geq 1$, the existence of $k$-uniform IASI is established for a bipartite graph. Now recall the Theorem 1.7 that a connected graph $G$ admits a 2-uniform IASI if and only if it is bipartite. Hence no non-bipartite graphs can have a 2-uniform IASI. Then, what are the admissible values of $k \neq 2$, for a non-bipartite graph to have a $k$-uniform IASI? The following results settle this problem. 
Theorem 2.15 Let $k$ be a positive integer. If $G$ is a graph which admits a $k$-uniform IASI, then any subgraph $H$ of $G$ also admits the same $k$-uniform IASI.

Proof: Let $G$ be a graph which admits a $k$-uniform IASI and $H$ be a subgraph of $G$. Let $f^{*}$ be the restriction of $f$ to $V(H)$. Then $g_{f^{*}}$ is the corresponding restriction of $g_{f}$ to $E(H)$. Then clearly, $f^{*}$ is a set-indexer on $\mathrm{H}$. This set-indexer may be called the induced set-indexer on $H$. Since $g_{f}(e)=k$ for all $e \in E(G)$, we have $g_{f^{*}}(e)=k$ for all $e \in E(H)$. Hence $H$ admits $k$-uniform IASI.

Remark 2.16 As the contrapositive statement of the above theorem, we observe that if a graph $G$ does not have a $k$-uniform IASI, then any supergraph of $G$ does not admit a $k$-uniform IASI.

Theorem 2.17 A complete graph $K_{n}$ admits a $k$-uniform IASI for positive odd integer $k$.

Proof: Let $\left\{v_{1}, v_{2}, v_{3}, \cdots, v_{n}\right\}$ be the vertex set of the complete graph $K_{n}$. Let $d$ and $m$ be a positive integer. Assign the set $\{i, i+d, i+2 d, \cdots, i+(m-$ 1) $d\}, i \leq i \leq n$ to each vertex $v_{i}$. Then an edge $v_{i} v_{j}$ of $K_{n}$ has the indexing set $\{i+j, i+j+d, i+j+2 d, \cdots, i+j+2(m-1) d\}$. That is, the set-indexing number of $v_{i} v_{j}$ is $2 m-1$. Since $v_{i}$ and $v_{j}$ are arbitrary vertices of $K_{n}$, all edges of it has the set-indexing number $2 m-1$. Then $K_{n}$ admits a $k$-uniform IASI, where $k=2 m-1$.

Remark 2.18 Being a subgraph of a complete graph $K_{n}$, any connected graph $G$ admits a $k$-uniform IASI for all positive odd integers $k$.

Hence the interest of studying the graphs which admit $k$-uniform IASI may be related to an even integer $k>2$. Hence what are these graphs which admit $k$-uniform IASI for even integer $k$, other than bipartite graphs? The following theorem settles this problem.

Theorem 2.19 Let $G$ be a connected non-bipartite graph. Then $G$ admits a $k$-uniform IASI if and only if $k$ is a positive odd integer.

Proof: Necessary part of the result follows from Remark 2.18. Let $m, n$ be two positive integers. Let $G=\left(X_{1}, X_{2}\right)$ be a bipartite graph. Assign $m$ element sets to the vertices of $X_{1}$ and $n$-element sets to the vertices of $X_{2}$ as in Theorem 2.11 so that every edge of $G$ has the set-indexing number $k=$ $m+n-1$. Draw an edge between two vertices of $X_{1}$ (or $\left.X_{2}\right)$ that gives a minimal connected non-bipartite graph with given number of vertices. Without loss of generality, let $e=v_{i} v_{j}$ where $v_{i}, v_{j} \in X_{1}$ (or $e=v_{l+i} v_{l+j} ; v_{l+i}, v_{l+j} \in X_{2}$. Let 
$G_{1}=G \cup e$. If possible, assume that $G_{1}$ admits a $k$-uniform IASI where $k>2$ is an even integer. Then, the set-indexing number of $e$ is $2 m-1$ (or $2 n-1$ ). Since $G_{1}$ admits a $k$-uniform IASI, $k>2$, we have $m+n-1=2 m-1=2 n-1=k$, which is possible on when $m=n$, a contradiction. Hence $k$ can not be even. Hence, for a positive even integer $k$, by Remark 2.16, no supergraph of $G_{1}$ (including $K_{n}$ ) admits a $k$-uniform IASI for a positive integer $k$.

Hence, a graph $G$ admits a $k$-uniform IASI, for a positive even integer $k>2$ if and only if $G$ is bipartite. More generally,

Theorem 2.20 A graph $G$ admits a $k$-uniform IASI, for a positive integer $k$, if and only if $k$ is odd or $G$ is bipartite.

\section{References}

[1] B D Acharya, Set-Valuations and Their Applications, MRI Lecture notes in Applied Mathematics, The Mehta Research Institute of Mathematics and Mathematical Physics, New Delhi, 1983.

[2] T M K Anandavally, A Characterisation of 2-Uniform IASI Graphs, Int. Journal of Contemp. Math. Sciences, 8(10)(2013), 459-462.

[3] J A Bondy and U S R Murty, Graph Theory, Springer, 2008.

[4] J A Gallian, A Dynamic Survey of Graph Labelling, The Electronic Journal of Combinatorics (DS 16), 2011.

[5] K A Germina, Set-Valuations of a Graph and Applications, Final Technical Report, DST Grant-In-Aid Project No.SR/S4/277/05, The Dept. of Science and Technology (DST), Govt. of India, 2011.

[6] K A Germina and T M K Anandavally, Integer Additive Set-Indexers of a Graph:Sum Square Graphs, Journal of Combinatorics, Information and System Sciences, 37(2-4)(2012), 345-358.

[7] F Harary, Graph Theory, Addison-Wesley Publishing Company Inc., 1969.

[8] D B West, Introduction to Graph Theory, Pearson Education Inc., 2001.

Received: October 9, 2013 